\title{
Convective flow driven by a chemical nanopump
}

\author{
Yang Ding $\odot^{*}$ \\ Department of Chemical Engineering and Biotechnology, University of Cambridge, \\ Cambridge CB3 OAS, United Kingdom
}

Julyan H. E. Cartwright $\odot$

Instituto Andaluz de Ciencias de la Tierra, CSIC-Universidad de Granada, E-18100 Armilla, Granada, Spain and Instituto Carlos I de Física Teórica y Computacional, Universidad de Granada, E-18071 Granada, Spain

Silvana S. S. Cardoso 1

Department of Chemical Engineering and Biotechnology, University of Cambridge, CB3 OAS Cambridge, United Kingdom

\section{(Received 3 April 2019; accepted 24 June 2020; published 10 August 2020)}

\begin{abstract}
We demonstrate experimentally and theoretically that self-assembled precipitate membranes with dual permeability can initiate and maintain exchange nanoflows using the chemical-potential gradient of a dissolving solute. Moreover, we show how such chemical energy can drive stable, oscillatory, and explosive convective motions.
\end{abstract}

DOI: 10.1103/PhysRevFluids.5.082201

\section{INTRODUCTION}

Porous membranes are important in both nature and industry owing to their ability to control the exchange of chemicals between two distinct environments $[1,2]$. Examples include hydrothermal vent structures [3,4], biological cell walls [5,6], and porous materials for water purification [7-9]. The simplest membranes are permeable and allow the exchange of chemicals in both directions. A more complex control of the flow is achieved by semipermeable membranes, which may allow some chemicals through but not others [10-13]. This control is normally the result of size restriction or electrical-charge effects; e.g., a small solvent molecule can move through the pores, but a larger solute is rejected. This latter class of membranes is of interest for selectivity in transport processes $[14,15]$. Only the most complex membranes exert selective transport in both directions, such as biological ones [16,17].

Facilitated diffusion of a solute across a membrane has been studied for a few decades now [18-20]. The solute molecule can be transported against its concentration gradient by first reacting with a carrier molecule. The usual energy sources for this assisted diffusion are either the chemical reaction itself $[19,20]$ or the chemical gradient of a second solute [18]. However, a much faster pump, driving convective flow instead of a diffusive one, can be designed. Indeed, semipermeable membranes enable the gradient of chemical potential of a solute to be converted into kinetic energy of the solvent in a fluid mixture [21-24]. This energy conversion occurs during osmotic flow across the membrane: The change in momentum of the solute molecules rebounding at the wall of the membrane induces a force that drives the solvent toward the environment rich in solute [25]. Chemical-garden membranes have been demonstrated to exhibit the necessary semipermeability for osmotic pumping [26]. For example, a cobalt silicate membrane allows water molecules across it,

\footnotetext{
*yd263@cam.ac.uk
} 
but rebounds silicate and cobalt ions approaching its surface. Modern work on chemical gardens has shown that the ensuing fluid-flow patterns near the membrane are complex and produce a wide variety of membrane structures in three- [27-31], two- [32,33], and even one-dimensional [34,35] configurations. However, no study has hitherto focused on the convective flow across the membrane. Here, we show how such a study can pave the way for the design of new materials with dynamical control over the flow.

In this work, we construct and model a convective nanopump. We consider a closed semipermeable precipitate membrane with dual permeability: In the low permeability regions, the gradient in chemical potential of a dissolving solute drives the flow of small solvent molecules, while in the larger pores both solvent and solute ions are transported by pressure gradients. We show experimentally and theoretically that such a system can maintain an exchange flow between the internal and external environments using the chemical potential or free energy of the solute. Furthermore, different dynamical regimes are demonstrated, ranging from stable to oscillatory and explosive motions. We explore the implications of our findings for the control of nanoscale pumping.

\section{EXPERIMENTAL METHODS}

To study the behavior of a closed, dual-permeability membrane, experiments were performed using cobalt (II) chloride hexahydrate $\left(\mathrm{CoCl}_{2} \cdot 6 \mathrm{H}_{2} \mathrm{O}\right)$ as the metal salt to react with an aqueous solution of sodium silicate $\left(\mathrm{Na}_{2} \mathrm{SiO}_{3}\right)$ with concentrations in the range $0.10-2.0 \mathrm{M}$. The experiments were conducted in a horizontal Hele-Shaw cell, consisting of two transparent acrylic plates $(130 \times$ $100 \times 6 \mathrm{~mm}$ ) separated by a gap of $1.0 \mathrm{~mm}$, with underlying uniform lighting from an light-emitting diode (LED) lightbox. The $\mathrm{CoCl}_{2} \cdot 6 \mathrm{H}_{2} \mathrm{O}$ (Sigma-Aldrich) crystals were initially pressed into pellets with a diameter of $10 \mathrm{~mm}$ and thickness $1 \mathrm{~mm}$ by a KBR Port-A-Press ${ }^{\mathrm{TM}}$ Kit (International Crystal Laboratories) under an equivalent pressure of $110 \mathrm{MPa}$. A pellet was placed at the center of the HeleShaw cell and the $\mathrm{Na}_{2} \mathrm{SiO}_{3}$ solution was injected to fill the cell. The dynamics of each experiment was recorded by a Nikon D300s digital single-lens reflex (DSLR) camera $(4288 \times 2848$ pixels) with a Hoya circular polarizing lens filter, located above the cell. The motion in the silicate solution surrounding the pellet was visualized with seed polyamide particles with diameter of $20 \mu \mathrm{m}$ (Dantec Dynamics). An area of the cell of approximately $30 \mathrm{~mm}^{2}$ was photographed at a frame rate of $1 / 3 \mathrm{~Hz}$ to follow the particle trajectories. The measured inward and outward speeds near the membrane are given in Table I. Pressure measurements were made at the center of the cell (pressure sensor PS-2114, PASCO Scientific) at a frequency of $20 \mathrm{~Hz}$ and a resolution of $1 \mathrm{~Pa}$. The pressure and speed measurements were used to estimate the membrane permeabilities in the inflow and outflow regions, which are also given in Table I. The osmotic pressure $p_{o}$ across the semipermeable membrane was calculated based on the relation $[25,36,37] p_{o}=\phi_{\mathrm{Co}} c_{\mathrm{Co}} R T-\phi_{\mathrm{Si}} c_{\mathrm{Si}} R T$, where $\phi_{\mathrm{Co}}$ and $\phi_{\mathrm{Si}}$ are the activity coefficients for $\mathrm{CoCl}_{2}$ and $\mathrm{Na}_{2} \mathrm{SiO}_{3}$ solutions, respectively [38,39], $R$ is the ideal gas constant, and $T$ is the absolute temperature. A sample of the solution of cobalt within the pellet region was collected and its concentration was measured as $3.4 \mathrm{M}$ using an Agilent Cary 60 ultraviolet-visible spectrophotometer.

\section{RESULTS AND DISCUSSION}

The growth of the cobalt chloride membrane is shown in Fig. 1. Three zones can be distinguished: a central zone with a dark pink color is the cobalt chloride pellet, an intermediate liquid zone with color gradient from pink to clear contains the dissolved cobalt ion, $\mathrm{Co}^{2+}(\mathrm{aq})$; and the external zone with a purple color is the membrane, which contains precipitated cobalt silicate, $\mathrm{CoSiO}_{3}(\mathrm{~s})$. The motion of three polyamide seed particles is tracked in Fig. 2. The particles are seen to first approach the membrane surface and then move outward. Typical measure speeds for the inward and outward flows are given in Table I.

The flow in the vicinity of the membrane formed by reaction of two aqueous solutions, an inner solution containing cation $\mathrm{Co}^{2+}$ and an outer solution of anion $\mathrm{SiO}_{3}^{2-}$ at concentration $c_{\mathrm{Si}}$, 
TABLE I. Parameter values measured in the experiments and used in the linear and nonlinear models.

\begin{tabular}{|c|c|c|c|c|}
\hline Regime & & Explosive & Stable & Oscillatory \\
\hline$c_{\mathrm{Si}}(\mathrm{M})$ & $\begin{array}{c}\text { Measured } \\
\text { Nonlinear model }\end{array}$ & $\begin{array}{c}0.45+ \\
0.5\end{array}$ & $\begin{array}{c}0-0.25 \\
0.1\end{array}$ & $\begin{array}{c}0.25-0.45 \\
0.33\end{array}$ \\
\hline$p_{o}(\mathrm{MPa})$ & Nonlinear model & 14.03 & 14.69 & 14.46 \\
\hline$k_{\text {in }}\left(10^{-19} \mathrm{~m}^{2}\right)$ & $\begin{array}{c}\text { Measured } \\
\text { Nonlinear model }\end{array}$ & $\begin{array}{c}4.6-15 \\
5.9\end{array}$ & $\begin{array}{c}5.3-14 \\
12\end{array}$ & $\begin{array}{c}18-27 \\
21\end{array}$ \\
\hline$k_{\text {out }}\left(10^{-13} \mathrm{~m}^{2}\right)$ & $\begin{array}{c}\text { Measured } \\
\text { Nonlinear model }\end{array}$ & $\begin{array}{c}16-18 \\
18\end{array}$ & $\begin{array}{c}2.8-3.5 \\
3.5\end{array}$ & $\begin{array}{c}1.8-4.2 \\
1.5\end{array}$ \\
\hline $\begin{array}{l}u_{\text {in }}\left(\mu \mathrm{m} \mathrm{s}^{-1}\right) \\
u_{\text {out }}\left(\mu \mathrm{m} \mathrm{s}^{-1}\right)\end{array}$ & $\begin{array}{l}\text { Measured } \\
\text { Measured }\end{array}$ & $\begin{array}{c}14-17 \\
0-26\end{array}$ & $\begin{array}{c}9.5-14 \\
15-23\end{array}$ & $\begin{array}{l}6.8-18 \\
8.9-19\end{array}$ \\
\hline$N$ & Linear/nonlinear models & 0.079 & 2.7 & 4.7 \\
\hline$M$ & Linear/nonlinear models & 0.061 & 0.42 & 2.5 \\
\hline$c_{i}(\mathrm{M})$ & Nonlinear model & 0 & 0 & 0.31 \\
\hline$p_{i}(\mathrm{kPa})$ & Nonlinear model & 0 & 0 & 0.40 \\
\hline$L_{m}(\mathrm{~mm})$ & $\begin{array}{c}\text { Measured } \\
\text { Nonlinear model }\end{array}$ & $\begin{array}{c}0-2.0 \\
1.0\end{array}$ & $\begin{array}{c}0-3.0 \\
0.70\end{array}$ & $\begin{array}{c}0-3.0 \\
2.4\end{array}$ \\
\hline
\end{tabular}

is depicted in Figs. 3(a) and 3(b). The initial membrane is impermeable to both the cobalt and silicate ions, but permeable to water molecules. As a result, a gradient of osmotic pressure $p_{o} / L_{m}$ drives water from the exterior environment into the cell enclosed by the membrane, thus increasing the internal pressure $p$; here, $L_{m}$ is the membrane thickness. This increase in pressure, in turn, opens small cracks in the membrane and drives the saturated aqueous solution of cobalt outward through these. A dual permeability membrane is therefore formed: The chemistry controls the low permeability of the inflow regions, while the internal pressure and solid mechanics of the membrane determine the higher permeability in the outflow regions. At the outer surface of the membrane, the reaction

$$
\mathrm{Co}^{2+}(\mathrm{aq})+\mathrm{SiO}_{3}^{2-}(\mathrm{aq}) \rightarrow \mathrm{CoSiO}_{3}(\mathrm{~s})
$$

occurs at the interface between the two fluids, to form a precipitate layer of product, whose thickness grows with time. This nucleation and precipitation process is rapid [40,41], so that the formation of product is limited by the supply of silicate ion when $c_{\mathrm{Co}} \gg c_{\mathrm{Si}}$. Imaging and speed measurements show that the inflow and outflow regions of the membrane are distinct, and the permeability for the inflow is much smaller than that for the outflow, $k_{\text {in }} \ll k_{\text {out }}$ (see Table I); this complex structure enables the membrane to self-regulate both flows.

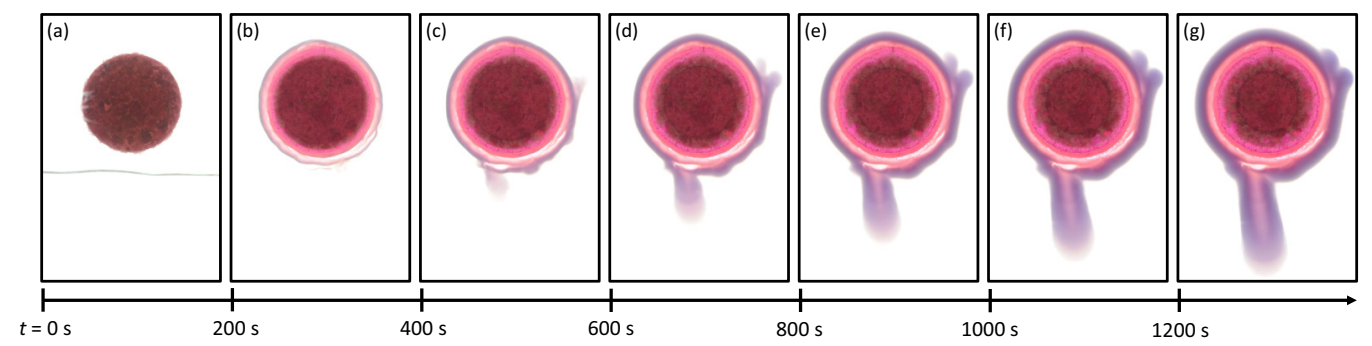

FIG. 1. Sequence of photographs showing the growth of the cobalt chloride membrane. Field of view: $17.48 \times 26.22 \mathrm{~mm}^{2}$. 

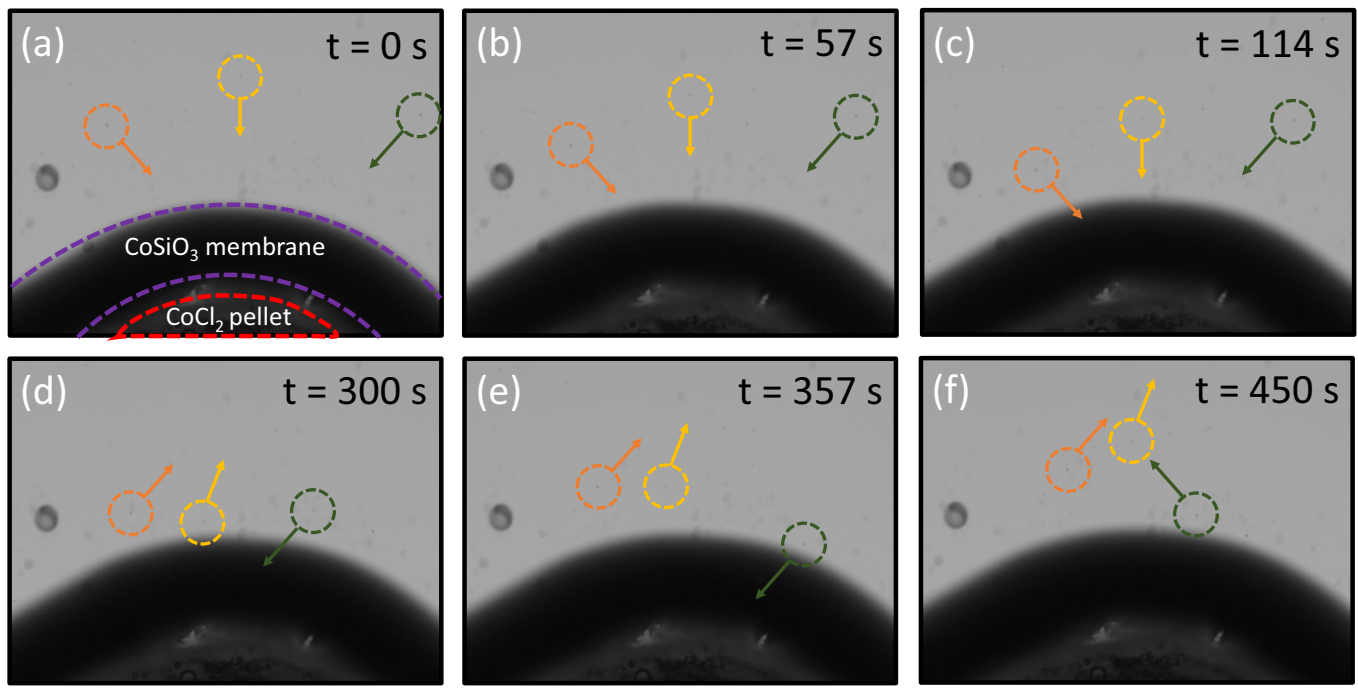

FIG. 2. Sequence of photographs showing the movement of three seed particles. The particles are in the middle of the dashed circles. Field of view: $8.74 \times 6.55 \mathrm{~mm}^{2}$.

In a simplified treatment, in which the membrane thickness is frozen in time, the system may be described by a reaction-advection equation for the transport of chemicals and formation of the precipitate, coupled with a pressure-volume balance. The concentration of product at the interface between the two fluids, $c$, and the relative pressure inside the membrane, $p$, evolve with time according to

$$
\begin{aligned}
& \frac{d c}{d t}=\left(u_{\text {in }} \frac{c_{\mathrm{Si}}}{L_{r}}-u_{\text {out }} \frac{c}{R}\right) H\left[u_{\text {out }}\right], \\
& \frac{d p}{d t}=\gamma\left(u_{\text {in }} A_{\text {in }}-u_{\text {out }} A_{\text {out }}\right) .
\end{aligned}
$$

Here, $u_{\text {in }}$ and $u_{\text {out }}$ are the radial speeds of external fluid towards the membrane and of the internal fluid at the outer edge of the membrane, respectively. The scale of the pore radii for the outflow is $R$ and reaction occurs over a length scale $L_{r}$ on the surface of the membrane. The Heaviside step function $H\left[u_{\text {out }}\right]$ describes that the product is only formed when there is outflow of cobalt ions. We use the coefficient $\gamma=(\partial p / \partial V)_{T}=2 L_{m} E /\left(R_{p} V_{p}\right)$ to describe the deformation of the thin cylindrical precipitate membrane; here $R_{p}$ and $V_{p}$ are the radius and volume enclosed by the cylindrical membrane, while $E$ denotes Young's modulus for the membrane material. The surface areas for inward osmotic flow $A_{\text {in }}$ and for the outward flow $A_{\text {out }}$ are assumed constant. The first equation describes a balance between the formation of product by reaction and its dilution owing to the spreading of the outflow. The second equation quantifies the change in pressure owing to the change in the volume of fluid inside the cell and the deformation of the membrane.

The speeds of the radial inflow and outflow across the membrane are given by

$$
\begin{aligned}
u_{\mathrm{in}} & =\frac{k_{\mathrm{in}}}{\mu} \frac{p_{o}-p}{L_{m}}, \\
u_{\mathrm{out}} & =\frac{k_{\mathrm{out}}}{\mu} \frac{p}{L_{m}}\left(1-\frac{c}{c^{*}}\right) H\left[1-\frac{c}{c^{*}}\right],
\end{aligned}
$$

where $k_{\text {in }}$ and $k_{\text {out }}$ are the permeabilities $[25,36,42]$ of the membrane in the regions of inflow and outflow, respectively, and $\mu$ is the viscosity of the fluids without product. The dependence of the 
(a)

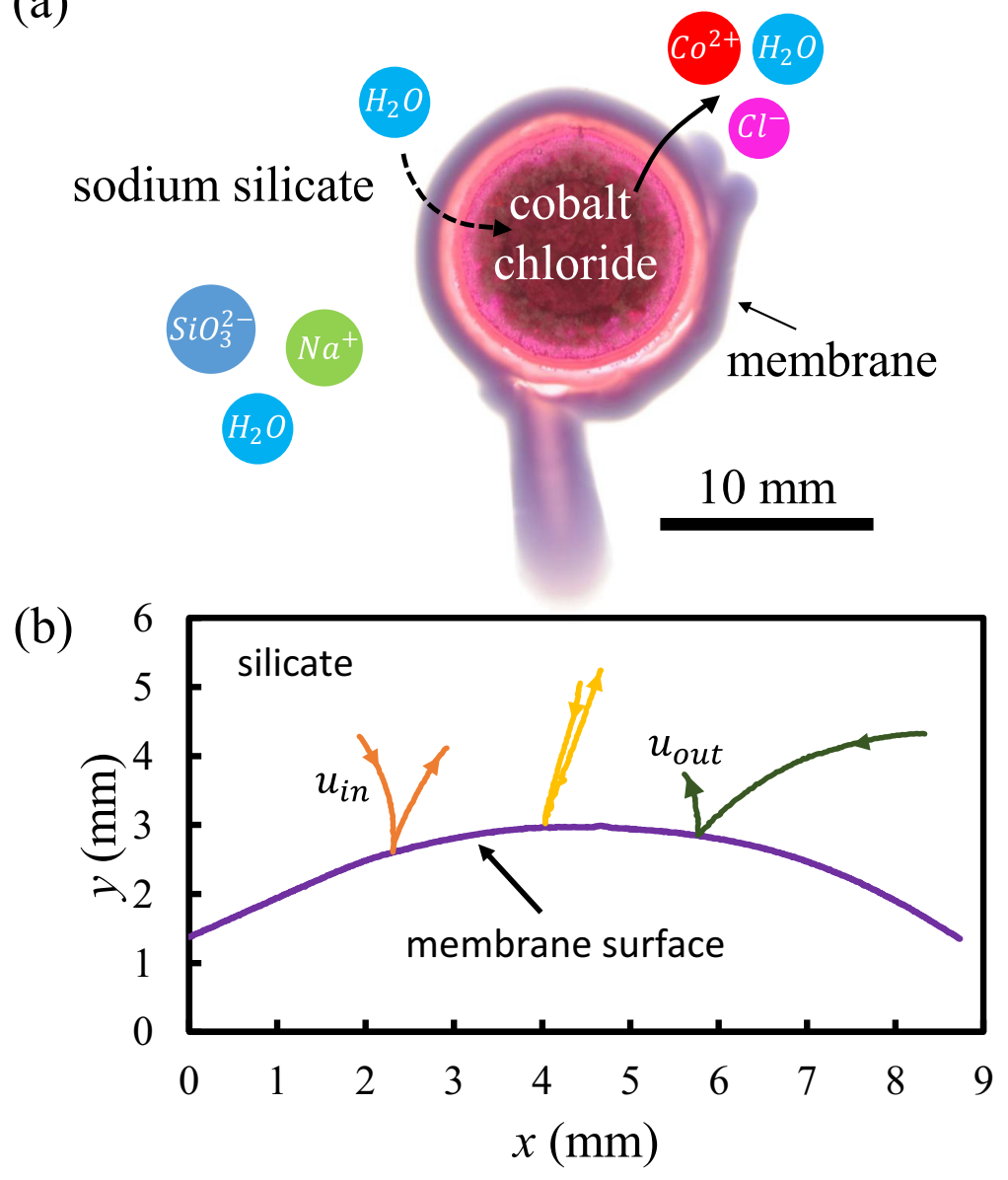

FIG. 3. (a) Photograph of the cobalt chloride pellet surrounded by the precipitate membrane and (b) measured trajectories of three seed particles near the membrane surface, at a silicate concentration of $0.28 \mathrm{M}$. The outside silicate solution flows toward the membrane with speed $u_{\text {in }}$, while the cobalt solution flows out of the membrane at speed $u_{\text {out }}$. The inflow and outflow regions on the surface of the membrane are closely located.

outflow speed on concentration of product at the interface of the inner and outer fluids was first proposed by Wagatsuma et al. [43] for the growth of a precipitate filament: As the concentration of product increases, the speed decreases, and ceases above the critical product concentration $c^{*}$. We introduce the Heaviside step function $H\left[1-c / c^{*}\right]$ to ensure that $c \leqslant c^{*}$ and $u_{\text {out }} \geqslant 0$.

Substituting the speed relations (3) into the governing equations (2) and nondimensionalizing using the scales for time $t_{s}=\mu R_{p} V_{p} /\left(2 E A_{\text {out }} k_{\text {out }}\right)$, pressure $p_{s}=2 E L_{m} A_{\text {out }} R /\left(V_{p} R_{p}\right)$, and concentration $c_{s}=c^{*}$ leads to the following simplified coupled equations:

$$
\begin{aligned}
& \frac{d \hat{c}}{d \hat{t}}=\left[M+\left(-\hat{c} \hat{p}+\hat{c}^{2} \hat{p}\right)\right] H[1-\hat{c}], \\
& \frac{d \hat{p}}{d \hat{t}}=N+(-\hat{p}+\hat{c} \hat{p}) H[1-\hat{c}] .
\end{aligned}
$$

We have assumed here that $p_{o} \gg p$ for our system. The evolution of the system depends therefore on two nondimensional groups only: $N=p_{o} k_{\text {in }} A_{\text {in }} V_{p} R_{p} /\left(2 E L_{m} k_{\text {out }} A_{\text {out }}^{2} R\right)$ is proportional to the 

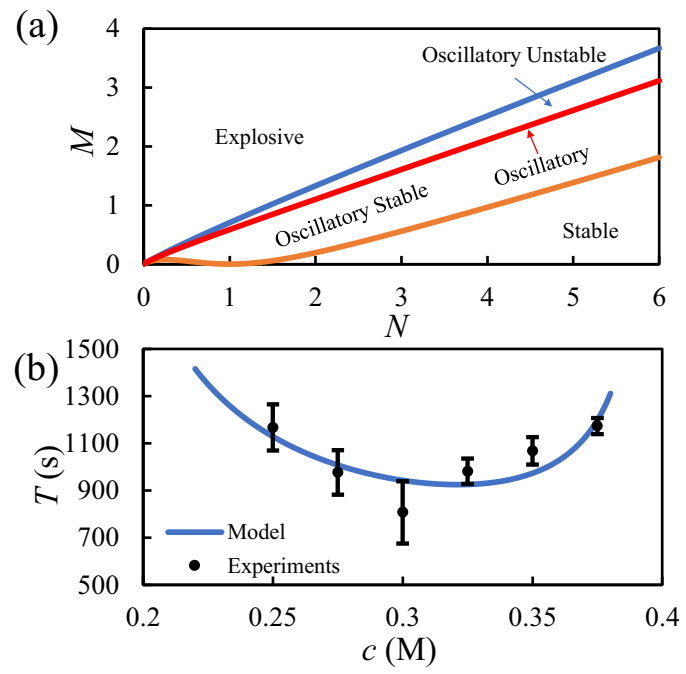

FIG. 4. (a) Regime diagram showing the regions of the parameter space where the system is stable, oscillatory, and explosive. $N$ is proportional to the osmotic pressure, while $M / N$ is proportional to the silicate solution concentration. (b) Period of oscillation as a function of the concentration of the silicate solution in the oscillatory regime. Shown are the experimental measurements and the prediction of Eq. (5).

concentration of silicate ion and represents a nondimensional rate for accumulation of solid; and $M=N\left[\left(c_{\mathrm{Si}} A_{\text {out }} R /\left(c^{*} A_{\text {in }} L_{r}\right)\right]\right.$ is proportional to the osmotic pressure, and hence the cobalt ion concentration, and represents nondimensional osmotic pumping rate. In the simplified limit of $A_{\text {out }} \approx A_{\text {in }}$ and $R / L_{r} \approx 1$, then $M \approx N c_{\mathrm{Si}} / c^{*}$.

Linear stability analysis of the governing equations (4) around the steady-state product concentration $\hat{c}_{s s}=M / N$ and internal pressure $\hat{p}_{s s}=N^{2} /(N-M)$ allows the identification of several dynamic behaviours for different concentrations of silicate and cobalt. Figure 4 illustrates the stable, oscillatory, and explosive regimes. At low concentration of silicate (low $M$ ), the system is stable: The internal fluid pressure and the concentration of product decrease with time. At higher concentrations of silicate, the system becomes oscillatory, exhibiting damped, stable, and growing oscillations of pressure and concentration as the concentration of silicate increases. The system develops a rapid, explosive pressure increase at still larger concentrations of silicate (high $M$ ). We should note that the identification of these regimes of behavior and the parameter ranges over which they exist is done nondimensionally, so that it is valid for any other chemical system involving an osmotic membrane with the same transport mechanisms.

In the oscillatory regime, we expect the period of oscillation to follow

$$
T=2 \pi t_{s}\left\{N-M-\frac{\left[M^{2}-2 M N(1+N)+N^{2}(1+N)\right]^{2}}{4(M-N)^{2} N^{2}}\right\}^{-1 / 2} .
$$

Figure 4 shows a comparison of this theoretical prediction with our experimental measurements for $N=4.7, t_{s}=223 \mathrm{~s}$, and $c^{*} A_{\text {in }} L_{r} /\left(A_{\text {out }} R\right)=0.64 \mathrm{M}$. As expected, the oscillatory regime develops in a window of intermediate concentrations of silicate, outside which the period of oscillation quickly grows to infinity. These system parameters allow us to deduce the following properties for the membrane: $E A_{\text {out }}=7.4 \times 10^{-3} \mathrm{~Pa} \mathrm{~m}^{2}, A_{\text {in }} / A_{\text {out }}=0.98$, and $R=4.5 \times 10^{-4} \mathrm{~m}$. Below, we use these properties to determine the nonlinear time-dependent behavior of the system through numerical integration of (4).

In the laboratory, we can measure the surface area of the solid membrane $S$ but not the concentration of solid at the reaction front on the outer surface of the membrane. However, we 


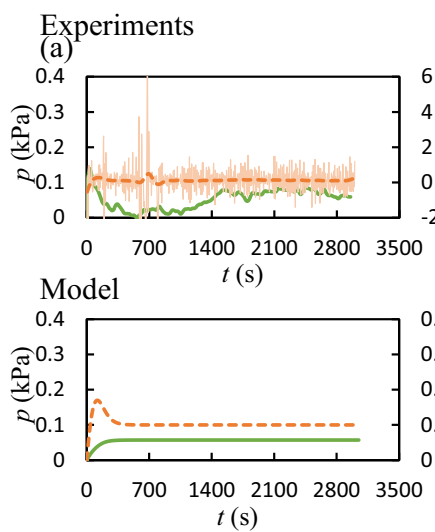

(b)

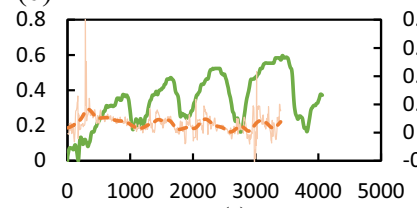

$t(\mathrm{~s})$

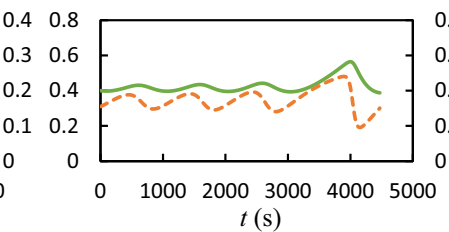

(c)
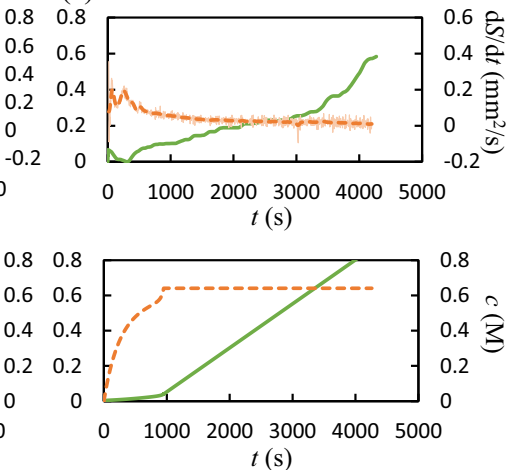

FIG. 5. Experimental measurements (top) and theoretical predictions (bottom) for the membrane behavior in the (a) stable, (b) oscillatory, and (c) explosive regimes, for constant membrane permeabilities and thickness (see Table I). Shown are the experimental pressure (solid line) and rate of increase of surface area of precipitate (dashed line), as well as the theoretical predictions of (5) for the pressure (solid line) and concentration of product (dashed line).

can relate these two quantities give that the surface area grows according to $d S / d t \sim d L_{m} / d t \sim$ $u_{\text {out }} c / \rho_{s} \sim\left(-d c / d t+u_{\text {in }} c_{\mathrm{Si}} / L_{r}\right) / \rho_{s}$, where $\rho_{s}$ is the bulk density of the solid precipitate. The last term in brackets is approximately constant, so that any temporal oscillations in $c$, and thus $\rho_{s}$, will be reflected as oscillations in $S$ too. Figure 5 depicts the evolution of the measured rate of change of the surface area of the solid membrane, as well as the pressure inside the pellet, for a range of concentrations of the silicate solution. Also shown are the corresponding predictions of our simple model, Eq. (4), for the pressure and concentration of product, using permeabilities of the membrane in the inflow and outflow regions within the measured range and appropriate initial conditions $p_{i}$ and $c_{i}$ (see Table I). In the stable regime, Fig. 5(a), the pressure and concentration of product both grow to the steady-state values. There is good agreement between the measured and predicted pressure increases; the almost constant concentration of product is also well predicted by the observed $d S / d t \sim 0$. The oscillatory case in Fig. 5(b) exhibits oscillations for both pressure and $d S / d t$, which are initially in antiphase but gradually slip into phase at larger time. This behavior and the oscillation period are well predicted by the theory. In both the stable and oscillatory cases, the concentration of product remains below the critical value at which the outflow stops. In contrast, in the explosive behavior depicted in Fig. 5(c), the concentration grows to its maximum value at a time of $\approx 1000 \mathrm{~s}$, after which the flow out of the membrane ceases and the pressure increases very rapidly. The experiments reveal a strong decrease of $d S / d t$ to zero as the membrane becomes blocked with precipitate, as expected.

We can verify and validate further the consistency of our dynamical theory, by comparing its predictions for the inflow and outflow with the measured speeds near the membrane surface. Figure 6 shows both the average and maximum predicted speeds, for the cases in the stable, oscillatory, and explosive regimes. The inward velocity is approximately uniform in the azimuthal direction, over the inflow regions of the membrane, but has a parabolic profile across the thin Hele-Shaw cell gap; the maximum prediction takes into account this effect. The outflow velocity is more localized in the azimuthal direction, with outward jetlike flows interspaced by regions of much slower flow; here, we take into account the centerline speed for a two-dimensional jet in a porous medium for the predicted maximum speed [42]. Given the simplicity of the model derived here, the agreement between measured and predicted speeds in Fig. 6 is excellent. The slight underprediction of the inward and outward speeds for the explosive case may be accounted for by the abrupt closure 
(a)

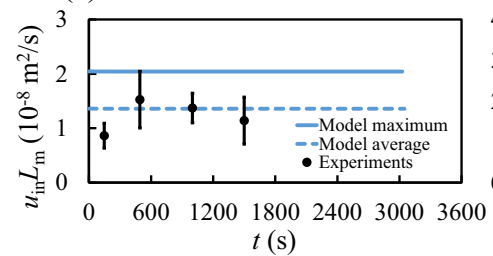

$t(\mathrm{~s})$

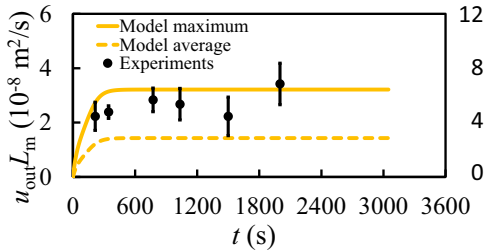

(b)
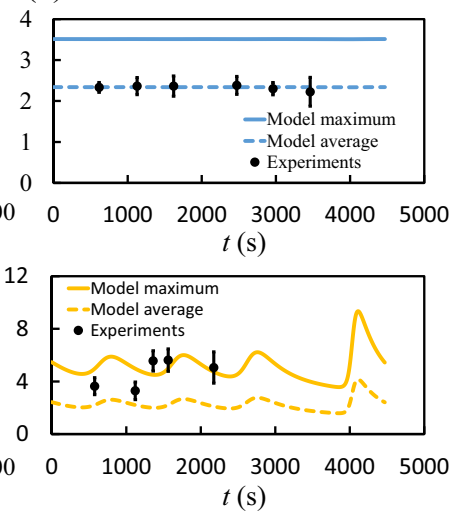

(c)
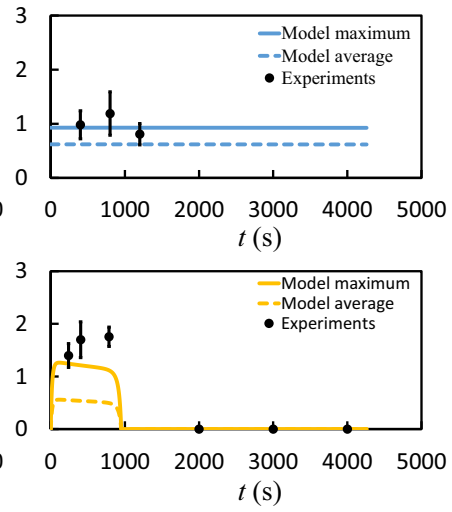

FIG. 6. Comparison of experimental measurements and theoretical predictions for the inflow (top) and outflow (bottom) speeds near the membrane surface in the (a) stable, (b) oscillatory, and (c) explosive regimes, for constant membrane permeabilities and thickness (see Table I). The theoretical predictions for both the average speed and the maximum speed, taking into account the flow profiles in the transverse and azimuthal directions, are shown.

of the membrane predicted by the model at $t=1000 \mathrm{~s}$, while the actual blocking of the membrane is more gradual.

We have demonstrated experimentally and theoretically that a precipitate membrane has the ability to self-regulate the inward and outward flows, so that they are of similar magnitude. It does so by developing regions with two very distinct permeabilities: The low permeability drives osmotic flow of solvent inward, while the larger permeability allows the outward flow of the solute solution. This quasibalance of flows enables the exchange through the membrane wall to be long lived. We have identified the emergent dynamical regimes leading to stable, oscillatory, and explosive flows. Our study shows how chemical energy of a solute can be converted into kinetic energy of both the solvent and the solute in a mixture, and thereby sustain an exchange flow between the internal and external environments. Given the genericity of these energy conversions, our results constitute a platform for the design of chemical nanopumps.

\section{ACKNOWLEDGMENTS}

J.H.E.C. acknowledges the financial support of the Spanish Ministry of Science, MINCINN Project No. FIS2016-77692-C2-2-P. The authors acknowledge the contribution of the COST Action No. CA17120.

[1] L. Buehler, Cell Membranes (Garland Science, New York, 2015).

[2] K. Li, Ceramic Membranes for Separation and Reaction (John Wiley \& Sons, New York, 2007).

[3] D. S. Kelley, J. A. Karson, D. K. Blackman, G. L. Früh-Green, D. A. Butterfield, M. D. Lilley, E. J. Olson, M. O. Schrenk, K. K. Roe, G. T. Lebon et al., An off-axis hydrothermal vent field near the mid-Atlantic ridge at $30 \mathrm{n}$, Nature (London) 412, 145 (2001).

[4] D. S. Kelley, J. A. Karson, G. L. Früh-Green, D. R. Yoerger, T. M. Shank, D. A. Butterfield, J. M. Hayes, M. O. Schrenk, E. J. Olson, G. Proskurowski, M. Jakuba, A. Bradley, B. Larson, K. Ludwig, D. Glickson, K. Buckman, A. S. Bradley, W. J. Brazelton, K. Roe, M. J. Elend, A. Delacour, S. M. Bernasconi, M. D. Lilley, J. A. Baross, R. E. Summons, and S. P. Sylva, A serpentinite-hosted ecosystem: The lost city hydrothermal field, Science 307, 1428 (2005). 
[5] M. S. Bretscher and M. C. Raff, Mammalian plasma membranes, Nature (London) 258, 43 (1975).

[6] E. R. Rojas, G. Billings, P. D. Odermatt, G. K. Auer, L. Zhu, A. Miguel, F. Chang, D. B. Weibel, J. A. Theriot, and K. C. Huang, The outer membrane is an essential load-bearing element in gram-negative bacteria, Nature (London) 559, 617 (2018).

[7] Y. Han, Z. Xu, and C. Gao, Ultrathin graphene nanofiltration membrane for water purification, Adv. Funct. Mater. 23, 3693 (2013).

[8] J. R. Werber, C. O. Osuji, and M. Elimelech, Materials for next-generation desalination and water purification membranes, Nat. Rev. Mater. 1, 16018 (2016).

[9] Z. Jiang, S. Karan, and A. G. Livingston, Thin films: Water transport through ultrathin polyamide nanofilms used for reverse osmosis, Adv. Mater. 30, 1870107 (2018).

[10] P. Silva, S. Han, and A. G. Livingston, Solvent transport in organic solvent nanofiltration membranes, J. Membr. Sci. 262, 49 (2005).

[11] A. V. Raghunathan and N. R. Aluru, Molecular Understanding of Osmosis in Semipermeable Membranes, Phys. Rev. Lett. 97, 024501 (2006).

[12] P. Marchetti, M. F. Jimenez Solomon, G. Szekely, and A. G. Livingston, Molecular Separation with Organic Solvent Nanofiltration: A Critical Review, Chem. Rev. 114, 10735 (2014).

[13] I. I. Ryzhkov, D. V. Lebedev, V. S. Solodovnichenko, A. V. Shiverskiy, and M. M. Simunin, InducedCharge Enhancement of the Diffusion Potential in Membranes with Polarizable Nanopores, Phys. Rev. Lett. 119, 226001 (2017).

[14] A. Kalra, S. Garde, and G. Hummer, Osmotic water transport through carbon nanotube membranes, Proc. Natl. Acad. Sci. USA 100, 10175 (2003).

[15] C. B. Picallo, S. Gravelle, L. Joly, E. Charlaix, and L. Bocquet, Nanofluidic Osmotic Diodes: Theory and Molecular Dynamics Simulations, Phys. Rev. Lett. 111, 244501 (2013).

[16] A. B. Pardee, Membrane transport proteins, Science 162, 632 (1968).

[17] W. Wickner and R. Schekman, Protein translocation across biological membranes, Science 310, 1452 (2005).

[18] E. Cussler, Membranes which pump, AIChE J. 17, 1300 (1971).

[19] B. Perrin, R. Couturier, C. Nigon, P. Michalon, and B. Maisterrena, Artificial enzymic membrane pump for glucose transport against its chemical gradient, J. Membr. Sci. 147, 95 (1998).

[20] C. Cheng, P. R. McGonigal, S. T. Schneebeli, H. Li, N. A. Vermeulen, C. Ke, and J. F. Stoddart, An artificial molecular pump, Nat. Nanotechnol. 10, 547 (2015).

[21] J. H. van't Hoff, Die Rolle des osmotischen Druckes in der Analogie zwischen Lösungen und Gasen, Z. Phys. Chem. 1, 481 (1887).

[22] Lord Rayleigh, The theory of solution, Nature (London) 55, 253 (1897).

[23] J. W. Gibbs, Semi-permeable films and osmotic pressure, Nature (London) 55, 461 (1897).

[24] U. Lachish, Osmosis and thermodynamics, Am. J. Phys. 75, 997 (2007).

[25] S. S. S. Cardoso and J. H. E. Cartwright, Dynamics of osmosis in a porous medium, Roy. Soc. Open Sci. 1, 140352 (2014).

[26] L. M. Barge, S. S. S. Cardoso, J. H. E. Cartwright, G. J. T. Cooper, L. Cronin, A. De Wit, I. J. Doloboff, B. Escribano, R. E. Goldstein, F. Haudin, D. E. H. Jones, A. L. Mackay, J. Maselko, J. J. Pagano, J. Pantaleone, M. J. Russell, C. I. Sainz-Díaz, O. Steinbock, D. A. Stone, Y. Tanimoto, and N. L. Thomas, From chemical gardens to chemobrionics, Chem. Rev. 115, 8652 (2015).

[27] S. Thouvenel-Romans and O. Steinbock, Oscillatory growth of silica tubes in chemical gardens, J. Am. Chem. Soc. 125, 4338 (2003).

[28] D. A. Stone and R. E. Goldstein, Tubular precipitation and redox gradients on a bubbling template, Proc. Natl. Acad. Sci. USA 101, 11537 (2004).

[29] J. Pantaleone, A. Toth, D. Horvath, J. R. McMahan, R. Smith, D. Butki, J. Braden, E. Mathews, H. Geri, and J. Maselko, Oscillations of a chemical garden, Phys. Rev. E 77, 046207 (2008).

[30] J. Pantaleone, A. Toth, D. Horvath, L. RoseFigura, W. Morgan, and J. Maselko, Pressure oscillations in chemical gardens, Phys. Rev. E 79, 056221 (2009).

[31] V. Kaminker, J. Maselko, and J. Pantaleone, The dynamics of open precipitation tubes, J. Chem. Phys. 140, 244901 (2014). 
[32] J. H. E. Cartwright, J. M. García-Ruiz, M. L. Novella, and F. Otálora, Formation of chemical gardens, J. Colloid Interface Sci. 256, 351 (2002).

[33] F. Haudin, J. H. E. Cartwright, F. Brau, and A. De Wit, Spiral precipitation patterns in confined chemical gardens, Proc. Natl. Acad. Sci. USA 111, 17363 (2014).

[34] B. C. Batista and O. Steinbock, Growing inorganic membranes in microfluidic devices: Chemical gardens reduced to linear walls, J. Phys. Chem. C 119, 27045 (2015).

[35] Y. Ding, B. Batista, O. Steinbock, J. H. E. Cartwright, and S. S. S. Cardoso, Wavy membranes and the growth rate of a planar chemical garden: Enhanced diffusion and bioenergetics, Proc. Natl. Acad. Sci. USA 113, 9182 (2016).

[36] O. Kedem and A. Katchalsky, Thermodynamic analysis of the permeability of biological membranes to non-electrolytes, Biochim. Biophys. Acta 27, 229 (1958).

[37] A. J. Staverman, The theory of measurement of osmotic pressure, Rec. Trav. Chim. Pays-Bas 70, 344 (1951).

[38] R. A. Robinson and R. H. Stokes, Electrolyte Solutions, 2nd ed. (Dover, New York, 2012).

[39] H. Park and P. Englezos, Osmotic coefficient data for $\mathrm{Na}_{2} \mathrm{SiO}_{3}$ and $\mathrm{Na}_{2} \mathrm{SiO}_{3}-\mathrm{NaOH}$ by an isopiestic method and modeling using Pitzer's model, Fluid Phase Equilib. 153, 87 (1998).

[40] P. Hirsch-Ayalon, Precipitate impregnated membranes: II, Recl. Trav. Chim. Pays-Bas 80, 365 (1961).

[41] P. Hirsch-Ayalon, Precipitation membranes, J. Membr. Biol. 12, 349 (1973).

[42] D. L. Turcotte and G. Schubert, Geodynamics (Cambridge University Press, Cambridge, UK, 2002).

[43] S. Wagatsuma, T. Higashi, Y. Sumino, and A. Achiwa, Pattern of a confined chemical garden controlled by injection speed, Phys. Rev. E 95, 052220 (2017). 